\title{
Toponym Recognition in Historical Maps by Gazetteer Alignment
}

\author{
Jerod Weinman \\ Grinnell College, Grinnell, Iowa, USA \\ weinman@grinnell.edu
}

\begin{abstract}
Historical map documents are increasingly digitized for widespread access, but most are only coarsely indexed with meta-data while the contents are largely unsearchable. We propose to increase searchability by automatically recognizing the place names in these digitized artifacts. Using a word recognition system that produces a noisy ranked list of initial hypotheses from a lexicon of viable toponyms, we form a joint probabilistic model for inferring the most likely latent alignment between image toponyms and a gazetteer of known place locations. After a robust generalized RANSAC algorithm identifies the global alignment, we rerank the toponym hypotheses by their posterior probability. Experiments demonstrate a significant boost in word recognition accuracy on a manually annotated set of 19th century U.S. state and regional maps.
\end{abstract}

Keywords-georectification, toponym recognition, historical maps, gazetteer, generalized RANSAC, MLESAC, correspondence

\section{INTRODUCTION}

Maps tell stories. They tell tales of politics, people, and progress. Important historical and print map collections have traditionally been available only to scholars and patrons who could access them physically. Fortunately, such artifacts are increasingly being digitized for wider access. For example, the John Carter Brown Library's online archive of Early American Images ${ }^{1}$ features over 10,000 maps from their physical collection of about 40,000, the David Rumsey Collection [1] contains 37,000, and the meta-collection OldMapsOnline.org collects thirteen institutions' holdings of nearly half a million maps in total.

Whether physical or digital, searching maps for an answer to a specific question could be laborious. The Library of Congress classification for maps includes only the map's region along with the subject, date, and source. ${ }^{2}$ Digitized holdings are only occasionally annotated with the geographical coordinates of their bounding box. Still, a researcher in pursuit of information about a very specific geographic place in a larger context may have to search visually through many artifacts, a time-consuming and error-prone process. Manually annotating so many artifacts is infeasible. While ventures such as Google Books make traditional print manuscripts searchable, traditional OCR techniques such as language modeling are unlikely to perform well on maps. As a result, these important information sources remain largely unsearchable.

By accurately transcribing the place names (toponyms) present on historical maps automatically, we aim to increase the accessibility of information locked in geographical

\footnotetext{
${ }^{1}$ See http://jcb.lunaimaging.com/luna/servlet/JCB 1 1

${ }^{2}$ See http://www.loc.gov/aba/cataloging/classification/lcco/lcco_g.pdf
}
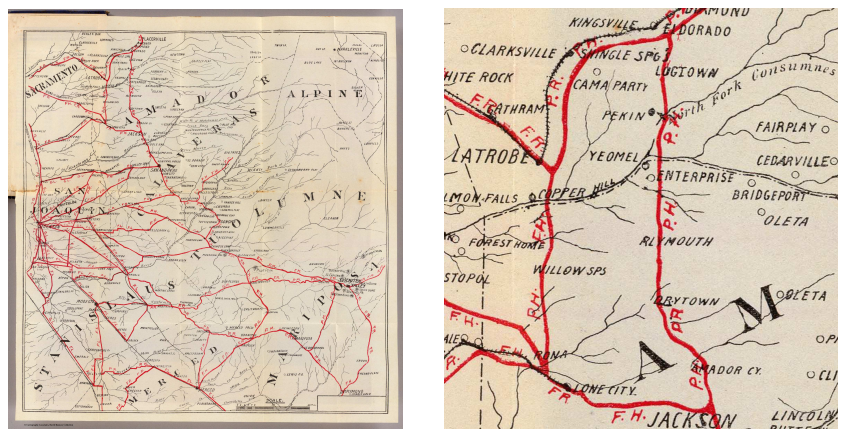

Figure 1. 1896 bicycle trail map of California's central valley and detail. (Image 1592006 @ Cartography Associates [1])

archives, enabling people to find and tell their stories. The task poses a significant technical challenge. As Figure 1 shows, map text can have curved baselines, arbitrary size and orientation, overlapping other features and text. Historical fonts and hand engraving further complicate recognition.

There is significant prior work on automated map processing: converting raster map images into a vectorized format, recognizing legend symbols, basic character/digit recognition in constrained domains such as cadastre (land parcel) maps. However, little work exists on applying general text recognition techniques to historical maps. In this paper we investigate three primary questions:

- How accurate is a general robust word recognition system on maps with minimal preprocessing?

- Can we align a map image to known geography using only noisy, uncertain word hypotheses?

- How much does a reasonable alignment improve the word hypotheses?

To facilitate geographical word recognition, we propose a joint probabilistic model for inferring an alignment between image toponyms and a gazetteer of known place locations. We introduce a generalized RANSAC algorithm for identifying a collection of correspondences from between pairs of candidate sets. Finally, we use the probability model and alignment to rerank the toponym hypotheses by their posterior probability. Section II reviews related work along with cartographical background information and our experimental data, a manually annotated set of 19th century U.S. state and regional maps. Section III describes our probabilistic model, search algorithm, and reranking calculation. Experiments in Section IV demonstrate positive answers to our three questions. Section V concludes, pointing to further work. 


\section{BACKGROUND}

\section{A. Related Work}

Separating text and graphics in map images is well studied [2], [3], and several works follow this separation by robust character recognition [4], [5], [6], [7], [8]. Most research focuses on modern, topographic, or cadaster maps, rather than historical maps. However, few have integrated geographical background knowledge with the recognition process. Gelbukh et al. [9] propose probability models for point, linear, and regional features, assuming the georectification (approximate correspondence between geographical coordinates and image pixels) is given. Using these models, they heuristically evaluate the likelihood of possible toponyms corresponding to a single OCR output and similar strings with small edit distance.

Our work makes two primary contributions. First, whereas Gelbukh et al. [9] assume georectified maps, we propose a robust search method for the coordinate correspondences. Second, rather than using a single OCR output, we rank many gazetteer words by their appearance probabilities, which are then used to calculate the final predicted word. Although text separation and word segmentation are non-trivial tasks, we assume word locations have already been correctly identified in order to assess an upper bound on how much geographical background might assist automated recognition.

\section{B. Cartography: Geoids and Projections}

Our intermediate goal is to determine a correspondence between coordinates in an image and coordinates on the earthgeorectification. Maps vary in their projections of spherical coordinates on the earth into planar Cartesian coordinates [10]. If the projection and "datum"- - the ellipsoid/geoid modeling the earth's shape - used to represent a particular map are unknown, georectification could be problematic.

Because the specific projection of any given map is often unknown, we choose a single projection family to use. The Hassler polyconic projection was frequently employed in the U.S. from its 1820 debut up through the 1950s [10, p. 124], and it requires only a central meridian and parallel. For these reasons-simplicity and historical popularity-we use the Hassler projection throughout this work. Our examination of 100 historical maps with scales ranging from the continental U.S., to smaller regions, individual states, and one city showed the most frequent projection family appeared to be conic. Although several models of the earth's shape exist, we use a simple spherical datum. Because our measurements (i.e., on the image) lack sufficient precision, other more appropriate models, such as the Clarke 1866 geoid, do not improve our results.

Given a correspondence between image coordinates and projected geographical locations, the two sets of coordinates are related by a simple planar transformation. If the projection and its parameters were known, a simple similarity transform (including scale, rotation, translation) suffices. However, errors either in our choice of projection family or its parameters force us to consider the affine space of Cartesian coordinate transforms, which allows the graticule lines (meridians and parallels) to intersect non-orthogonally via shearing. We find this added flexibility necessary to identify appropriate alignments.

\section{Data}

1) Gazetteer: The U.S. Board on Geographic Names (BGN) is part of the federal U.S. Geographical Survey created to "maintain uniform geographic name usage throughout the Federal Government". ${ }^{3}$ For the contiguous lower forty-eight states, the gazetteer of the USBGN Geographic Names Information System (GNIS) contains slightly over two million entries (called "features") in sixty five categories from Airport to Woods, though we use only Civil and Populated Place, which constitute the majority of our corpus toponyms. Each feature has primary geographical coordinates and is indexed by its state and county. The GNIS also contains historical features (i.e., municipalities that no longer exist) as well as alternate names (or spellings) for both existing and historical features.

2) Maps: For our experiments, we have annotated 12 maps from the David Rumsey collection [1], with each map's approximate region noted (i.e., the primary state or counties and any surrounding states having toponyms represented). A simple bound on coordinate ranges, as given by some online map collections, would also suffice to restrict the gazetteer ultimately used for recognition. Annotations in a simple XML format include tight piecewise linear baseline guides, text height and ground truth string for each toponym. ${ }^{4}$ In addition to the regional map shown in Figure 1, many are railroad and public survey maps. The average text height is $25 \pm 16$ pixels.
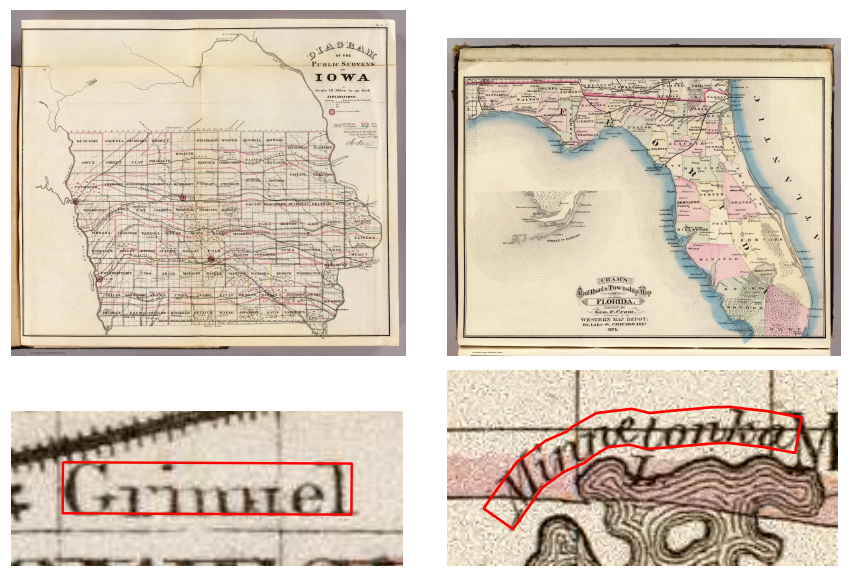

Figure 2. Top: Railroad and public survey maps. Bottom: Word bounding box annotations. (Image 1070007, 1070006, and 5370006 (C) Cartography Associates [1])

\section{APPROACH}

We define the joint probability over the recognized string for each word in the map along with its associated toponym (if it has one), the image coordinate of the geographical feature represented by the word, and the global georectification alignment, which relates image coordinates to the gazetteer of known feature locations projected into the same Cartesian plane. We search for a maximum marginal likelihood alignment with RANSAC and use the best alignment to update posterior probabilities for the recognized strings by marginalizing all other unknowns. The following subsections detail the model, search algorithm, and final inference calculation.

\footnotetext{
${ }^{3}$ U.S. Board on Geographic Names, http://geonames.usgs.gov

${ }^{4}$ Data available at http://hdl.handle.net/11084/3246.
} 


\section{A. Probability Model}

The dependencies among the observed and unknown values are given by the directed graphical model, a Bayesian network, shown in Figure 3 below.

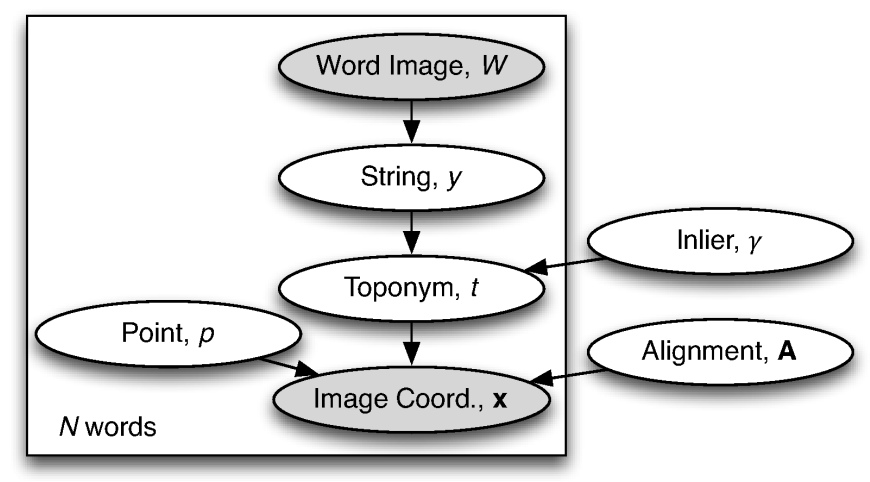

Figure 3. Graphical probability model (Bayesian network) for toponym recognition. Greyed ovals are observed values, and values inside the plate are replicated $N$ times, once for each word to be recognized in the map.

For a set of $N$ word images and image coordinates, we seek the strings, toponyms, points, and alignment that maximize the probability $P(\{y\},\{t\},\{p\}, \gamma, \mathbf{A} \mid\{W\},\{\mathbf{x}\})$ jointly described by the model above. For clarity we drop the subscript index $i$ from the values repeated for each word ( $W$, $y, t$, and $p$ ) when possible.

The probability of a recognized string hypothesis given the word image $P(y \mid W)$ can be given by any word recognition system that outputs string candidates and scores.

The inlier term $0 \leq \gamma \leq 1$ expresses the prior bias that a word image corresponds to a true toponym fitting the alignment model, versus some other non-geographical text. Thus, outliers from the alignment model take a null toponym, $t_{0}$ with probability $1-\gamma$, regardless of the string, so that

$$
P\left(t=t_{o} \mid y, \gamma\right)=1-\gamma
$$

We estimate the bias term $\gamma$ for each map via maximum likelihood during alignment search, described in section III-B.

The toponym probability for inliers is a simple ratio of counts; we assume no bias. Given a gazetteer containing a sequence of words for each unique geographical feature (i.e, "Box Butte County"), the probability is

$$
P(t \mid y, \gamma)=\gamma \frac{M(t, y)}{\sum_{t^{\prime}} M\left(t^{\prime}, y\right)},
$$

where $M(t, y) \in\{0,1\}$ indicates a match for word candidate $y$ in toponym $t$. Matches are case insensitive and include abbreviation expansions, so that the string $y=$ "St." would match a toponym containing "Saint" or "Street". To focus on identifying potential toponyms, we can marginalize over the finite set of strings given by the word recognizer,

$$
P(t \mid \gamma, W)=\sum_{y} P(t \mid y, \gamma) P(y \mid W) .
$$

The text for a toponym may be offset from the location predicted by the alignment. For example, while counties are typically labeled near their geographical centroid (the location given in our gazetteer), city labels appear to the side of their actual geographic location. We must therefore hypothesize several possibilities for the true point, given the location and geometry of the word in the image. To this end, the word's possible image coordinates include the centroid of the word bounding polygon, as well as several points along the perimeter as shown in Figure 4. With no bias for any of the points, the prior is uniform, $P(p) \propto 1$.

The predicted image coordinate is then a function of the toponym, the projection to Cartesian coordinate frame (described in Section II-B), and the unknown alignment. Each toponym has its own spherical coordinates, giving the latitude and longitude, which are then projected into the 2-D plane via function $\vec{C}(t)$. These planar coordinates are then aligned to the image via the unknown affine transformation matrix $\mathbf{A}$, so that the predicted point is $\mathbf{A} \vec{C}(t)$. Letting $\mathbf{x}_{p}$ be the $p$ th candidate point's observed image coordinate, its probability density is the isotropic normal/Gaussian likelihood

$$
P\left(\mathbf{x}_{p} \mid p, t, \mathbf{A}\right)=\mathcal{N}\left(\mathbf{x}_{p} ; \mathbf{A} \vec{C}(t), \mathbf{I} \sigma^{2}\right)
$$

when $t \neq t_{0}$. However, when the word is associated with the null toponym $t=t_{0}$, an alignment model outlier, the density is uniform over the image.

Finally, the prior probability for the alignment $\mathbf{A}$ is uniform over a map-specific range of values for the angle, scale, and shear parameters, described further in the next section.

\section{B. Model Inference: Alignment with Generalized RANSAC}

When the $\gamma$ and A nodes outside the plate in Figure 3 are observed, calculating the optimal values for the remaining nodes is straightforward because the $N$ words become conditionally independent. From the posterior we get the optimal non-null toponym and point for each word,

$$
\widehat{p}, \widehat{t}=\arg \max _{p, t} P\left(\mathbf{x}_{p} \mid p, t, \mathbf{A}\right) P(p) P(t \mid \gamma, W) .
$$

Calculating the optimal values for the transformation $\mathbf{A}$ and inlier probability $\gamma$ is not as easy. Although the graph is a polytree, inference in such mixed discrete/continuous models is generally intractable. Therefore we use a RANSAC variant called MLESAC [11] as a robust search method to find a probable alignment.

MLESAC uses the marginal likelihood of parameters estimated from a sample of hypothesized correspondences to rank possible solutions. Zhang and Kosecká [12] modify the sampling framework for situations when correspondence candidates are not one-to-one but one-to-many. In our problem, each word in the map might be identified by one of several possible toponyms and located at one of many candidate image coordinates. In this many-to-many correspondence problem, we have a pair of match sets for each word and the generalized RANSAC procedure must select one candidate from each. ${ }^{5}$

Estimating an affine transform requires three correspondences. First, three words are randomly selected; then a nonnull toponym and image point are selected for each word. Once

\footnotetext{
${ }^{5}$ Our implementation extends Marco Zuliani's RANSAC toolbox, https:// github.com/RANSAC/RANSAC-Toolbox.
} 
a word is chosen, the sampling probability for a particular toponym is simply the marginal $P(t \mid W)$-Eq. (3) with $\gamma=1$-while the point choice is the uniform $P(p)$.

We calculate the affine transformation $\widehat{\mathbf{A}}$ from the three pairs of corresponding image and toponym coordinates. Using a $\mathrm{QR}$ decomposition of $\widehat{\mathbf{A}}$ to identify the rotation angle, scale factors, and shearing, we reject any sample whose transformation is unreasonable with respect to our prior knowledge of the map. In particular, the angle must be within $20^{\circ}$ of vertical, shearing must be less than 15 , and the ratio of the two scale factors must be no larger than 1.2. Moreover, we bound the scale factor of each dimension to between one tenth and ten times the geometric mean of the ratio of the ranges of word image and projected gazetteer coordinates.

The original MLESAC algorithm searches for a model $M$ that maximizes the error $E$ likelihood mixture

$$
P(E \mid M)=P(E \mid \text { Inlier, } M) \gamma+P(E \mid \text { Outlier })(1-\gamma) \text {. }
$$

Here, the model ranking function is the marginal likelihood of observed points, given the alignment and inlier bias,

$$
\mathcal{R}(\gamma, \mathbf{A})=\sum_{i} \log P\left(\mathbf{x}_{i} \mid \gamma, \mathbf{A}, W_{i}\right)
$$

where

$P(\mathbf{x} \mid \gamma, \mathbf{A}, W)=\sum_{t} \sum_{p} P\left(\mathbf{x}_{p} \mid p, t, \mathbf{A}\right) P(p) P(t \mid \gamma, W)$

Once the transformation $\widehat{\mathbf{A}}$ is fixed, we must estimate the global parameter $\gamma$. Because this parameter does not require great precision, we simply find the optimum ranking over a reasonable number of discrete samples (here, 128),

$$
\widehat{\gamma}=\arg \max _{\gamma \in[0,1]} \mathcal{R}(\gamma, \widehat{\mathbf{A}})
$$

We continue the process of sampling, fitting, and ranking using the standard RANSAC iteration requirements.

\section{Posterior Re-Ranking}

Having estimated the inlier bias $\widehat{\gamma}$ and the alignment $\widehat{\mathbf{A}}$, we can update the word recognizer's prior probabilities by calculating the posterior probability of each string, which requires marginalizing over the unknown toponyms and associated image coordinate points:

$$
\begin{aligned}
P(y \mid W, \mathbf{x}, \widehat{\gamma}, \widehat{\mathbf{A}}) \propto & P(y \mid W) \sum_{t} P(t \mid y, \widehat{\gamma}) \times \\
& \sum_{p} P\left(\mathbf{x}_{p} \mid p, t, \widehat{\mathbf{A}}\right) P(p) .(10)
\end{aligned}
$$

In practice, the number of points and supported toponyms are both bounded and reasonably small, so the posterior calculation is a straightforward sum of a probability matrix for each string.

Figure 4 shows how spatial terms contribute to re-ranking.

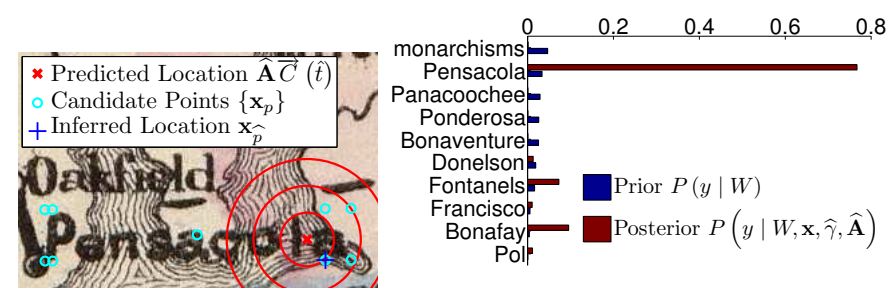

Figure 4. Relevant probabilities involved for the word "Pensacola". Left: Given the alignment $\widehat{\mathbf{A}}$, the predicted location of the best toponym $\widehat{t}=$ 2404503 (BGN Feature ID for "City of Pensacola") is shown at the mean of the likelihood, $\widehat{\mathbf{A}} \vec{C}(\widehat{t})$, surrounded by three isotropic standard deviations, $\sigma$. Right: prior and posterior probabilities for some top candidate strings.

\section{EXPERIMENTS}

In this section, we present answers to the questions posed in the introduction, noting initial recognition accuracy, alignment search robustness, and improved recognition accuracy using the alignment. Before evaluating the results, we detail the experimental setup and method of analysis.

\section{A. Method}

No text/graphics separation or line removal is used to preprocess the maps. Each annotated word image is normalized to a linear baseline by a thin plate spline. The normalized word image is given to a word recognition system [13], which produces a list of word hypotheses-lexical and non-lexicalwith their associated probabilities. We use both a standard American English lexicon of 82,000 words and a map-specific lexicon generated from the appropriate gazetteer. We tune the recognition model with words from four training maps; the remaining eight maps are held out for testing.

The inlier standard deviation $\sigma$ was determined from a set of training maps' ground truth. Having verified strings that uniquely identified toponyms, we use the resulting correspondences to estimate least-square affine transforms and measure the residual: $\sigma \approx 20$ pixels. Given the average map image size, a point is more likely to be an outlier (assuming $\gamma=0.5$ ) when it is more than about 88 pixels from its predicted location.

For each map, we must project the gazetteer's spherical coordinates into the image plane, which requires selecting a central meridian and parallel. For these, we use the centroid of the graticular area bounded by the map's gazetteer.

To identify an alignment of gazetteer coordinates with the word images, we prune the word string hypotheses given by the recognition system as follows. For each word we eliminate hypotheses comprising the bottom half of the cumulative probability, while preserving at least 2 , but no more than 5 candidates. These values are chosen based on the raw recognition system performance (described below). Even though many of the pruned strings support toponyms with a low chance of being selected for a RANSAC sample, this pruning speeds the search and removes many more outliers than inliers. Once the initial alignment is found, we use all original word hypotheses to calculate the posterior probability re-rankings. 
Table I. EXPERIMENTAL RESULTS: ERROR PERCENTAGES AND HARMONIC MEAN OF CORRECT WORDS' RANKS.

\begin{tabular}{|c|c|c|c|c|c|c|c|c|c|c|c|c|}
\hline \multirow[b]{3}{*}{ Map } & \multirow[b]{3}{*}{ Map } & \multirow{2}{*}{\multicolumn{3}{|c|}{ Num. Words }} & & & & & \multicolumn{4}{|c|}{ Among Words in Gaz. \& OCR Ranked } \\
\hline & & & & & \multicolumn{2}{|c|}{ Prior } & \multicolumn{2}{|c|}{ Posterior } & \multicolumn{2}{|c|}{ Prior } & \multicolumn{2}{|c|}{ Posterior } \\
\hline & & & & \& OCR & Word & Mean & Word & Mean & Word & Mean & Word & Mean \\
\hline Number & Region & Total & In Gaz. & Ranked & Error & Rank & Error & Rank & Error & Rank & Error & Rank \\
\hline 1592006 & Central Calif. & 286 & 162 & 146 & 72.03 & 2.529 & $73.51 \pm 0.17$ & $2.586 \pm 0.006$ & 59.59 & 1.594 & $61.13 \pm 0.33$ & $1.619 \pm 0.004$ \\
\hline 1070001 & Ohio & 261 & 220 & 185 & 56.32 & 1.865 & $44.64 \pm 0.81$ & $1.631 \pm 0.016$ & 40.00 & 1.356 & $23.24 \pm 1.23$ & $1.180 \pm 0.013$ \\
\hline 1070006 & Minnesota & 460 & 383 & 351 & 62.61 & 1.932 & $55.05 \pm 0.60$ & $1.771 \pm 0.011$ & 55.84 & 1.587 & $44.16 \pm 0.73$ & $1.425 \pm 0.009$ \\
\hline 1070007 & Iowa & 322 & 262 & 240 & 53.73 & 1.732 & $45.19 \pm 0.63$ & $1.591 \pm 0.010$ & 41.67 & 1.357 & $28.54 \pm 0.79$ & $1.227 \pm 0.008$ \\
\hline 5370026 & New Mexico & 210 & 134 & 124 & 54.76 & 1.894 & $55.36 \pm 0.53$ & $1.902 \pm 0.009$ & 38.71 & 1.357 & $39.52 \pm 0.99$ & $1.362 \pm 0.008$ \\
\hline 1070005 & Wisconsin & 216 & 189 & 159 & 58.33 & 1.87 & $52.55 \pm 0.95$ & $1.750 \pm 0.016$ & 45.91 & 1.439 & $37.42 \pm 1.51$ & $1.336 \pm 0.015$ \\
\hline 5370006 & Florida & 352 & 256 & 213 & 63.07 & 2.164 & $62.78 \pm 0.33$ & $2.167 \pm 0.008$ & 45.07 & 1.433 & $44.25 \pm 0.62$ & $1.429 \pm 0.007$ \\
\hline 1070010 & Arkansas & 218 & 194 & 167 & 64.68 & 2.044 & $51.61 \pm 1.16$ & $1.753 \pm 0.023$ & 54.49 & 1.580 & $37.43 \pm 1.52$ & $1.354 \pm 0.018$ \\
\hline & erall & 2325 & 1800 & 1585 & 60.99 & 1.983 & $55.43 \pm 0.26$ & $1.855 \pm 0.005$ & 48.26 & 1.465 & $39.18 \pm 0.35$ & $1.353 \pm 0.004$ \\
\hline
\end{tabular}

\section{B. Results}

To evaluate, we report the word error rates along with the harmonic mean of the correct word's rank in the probability distribution (whether prior or posterior), as summarized in Table I. For results depending on RANSAC, we give the average and standard error of several runs. The second column cluster ("Prior") reports the unaided performance of the general purpose robust word recognition system, which works surprisingly well for this task with no text/graphics separation. Although the correct string does not appear among the candidate hypotheses for $28 \%$ of test words, in the cases where it does appear, nearly $72 \%$ are within the top half of cumulative probability, $77 \%$ are rank 1 or 2 , and $89 \%$ are rank five or better. The overall character error, measured by edit distance, is $39.98 \%$. The training set has similar statistics.

After using the RANSAC algorithm to infer an alignment, we find the posterior probabilities (third column cluster, "Posterior") have eliminated 9\% of word errors, slightly boosting the overall mean rank. Character error also drops to $36.95 \%$. To test whether the noisy word hypotheses are actually yielding a reasonable alignment, we can instead start by giving only the ground truth words to RANSAC. Calculating posteriors of the OCR outputs using the resulting pseudo-ground truth alignment reduces error by about the same amount, indicating that our generalized RANSAC is robust to OCR noise in addition to toponym uncertainty.

Using the two feature categories we've chosen, only 1800 (77\%) of the words in the maps are present in the gazetteer. Moreover, some word images do not have the ground truth word among their ranked lists. Thus, nearly one third of the words cannot be improved through geography. Restricting our analysis to only those words present in the gazetteer and appearing somewhere in the OCR output (the last two column clusters), our method eliminates nearly $19 \%$ of word errors.

\section{Conclusions}

To recapitulate our three initial questions, robust word recognition works well enough on challenging map images for our RANSAC-based correspondence algorithm to determine an approximate georectification. By jointly inferring the most probable toponym hypotheses with the gazetteer alignment, we significantly reduce OCR errors.

After annotating additional maps, we plan to expand the gazetteer and probability model to include regional and linear features. We can also close the OCR loop by using the alignment to generate a more targeted gazetteer for recognizing individual word images so that early, internal hypothesis pruning is less likely to lower recall in the final candidate lists. Ultimately, we must integrate our recognition with automated text separation techniques. Although we have room for improvement, initial results are encouraging.

\section{ACKNOWLEDGMENTS}

The author gratefully acknowledges: R. Chande and D. Gumm, who curated and annotated maps; E. Carter, S. Danforth, N. Howe, S. Lillydahl, M. Nishimura, L. Tobias-Olsen, and B. Yoose for helpful conversations; J. Carl, C. Nicolson, and anonymous reviewers for suggestions on the manuscript.

\section{REFERENCES}

[1] Cartography Associates, "David Rumsey historical map collection." [Online]. Available: http://www.davidrumsey.com

[2] G. Myers, P. Mulgaonkar, C.-H. Chen, J. DeCurtins, and E. Chen, "Verification-based approach for automated text and feature extraction from raster-scanned maps," in Graphics Recognition, ser. LNCS, vol. 1072, 1996, pp. 190-203.

[3] H. Luo and R. Kasturi, "Improved directional morphological operations for separation of characters from maps/graphics," in Graphics Recognition, ser. LNCS, vol. 1389, 1998, pp. 35-47.

[4] L. Li, G. Nagy, A. Samal, S. Seth, and Y. Xu, "Cooperative text and line-art extraction from a topographic map," in Proc. ICDAR, 1999, pp. 467-470.

[5] A. Velázquez and S. Levachkine, "Text/graphics separation and recognition in raster-scanned color cartographic maps," in Graphics Recognition, 2003, pp. 63-74.

[6] J. Pouderoux, J.-C. Gonzato, A. Pereira, and P. Guitton, "Toponym recognition in scanned color topographic maps," in Proc. ICDAR, vol. 1, 2007, pp. 531-535.

[7] Y.-Y. Chiang and C. A. Knoblock, "An approach for recognizing text labels in raster maps," in Proc. ICPR, 2010, pp. 3199-3202.

[8] A. Pezeshk and R. L. Tutwiler, "Automatic feature extraction and text recognition from scanned topographic maps," IEEE Trans. on Geosci. and Remote Sens., vol. 49, no. 12, pp. 5047-5063, Dec. 2011.

[9] A. Gelbukh, S. Levachkine, and S.-Y. Han, "Resolving ambiguities in toponym recognition in cartographic maps," in Graphics Recognition, ser. LNCS, vol. 3088, 2004, pp. 75-86.

[10] J. P. Snyder, "Map projections: A working manual," U.S. Geological Survey, Washington, D.C., Tech. Rep. USGS Professional Paper 1395, 1987.

[11] P. H. S. Torr and A. Zisserman, "MLESAC: a new robust estimator with application to estimating image geometry," Comp. Vis. and Image Understanding, vol. 78, no. 1, pp. 138-156, Apr. 2000.

[12] W. Zhang and J. Kosecká, "Generalized RANSAC framework for relaxed correspondence problems," in Proc. Intl. Symp. 3D Data Proc., Visualization, and Transmission, 2006, pp. 854-860.

[13] J. Weinman, Z. Butler, D. Knoll, and J. Feild, "Toward integrated scene text reading," IEEE Trans. on Pattern Anal. Mach. Intell., 2013, to be published. 